\title{
Raman spectroscopy investigation of magnetite nanoparticles in ferrofluids
}

\author{
L. Slavov a,d,*, M.V. Abrashev ${ }^{b}$, T. Merodiiska ${ }^{a}$, Ch. Gelev $^{a}$, R.E. Vandenberghe ${ }^{c}$, I. Markova-Deneva $^{\mathrm{d}}$, \\ I. Nedkov ${ }^{a}$ \\ a Institute of Electronics, Microwave Magnetics, BAS, 72, Tzarigradsko Chaussee, 1784 Sofia, Bulgaria \\ ${ }^{\mathrm{b}}$ Faculty of Physics, University of Sofia, 1164 Sofia, Bulgaria \\ c Department of Subatomic and Radiation Physics, University of Gent, B-9000 Gent, Belgium \\ d Department of Non-Ferrous Metals and Semiconductor Technologies, University of Chemical Technology and Metallurgy, 8 Kliment Ohridsky Blvd., 1756 Sofia, Bulgaria
}

\section{A R T I C L E I N F O}

\section{Article history:}

Received 15 May 2009

Received in revised form

22 December 2009

\section{Keywords:}

Magnetite nanoparticles

Ferrofluids

Raman spectroscopy

FTIR spectroscopy

Cyclodextrin

Superparamagnetism

\begin{abstract}
A B S T R A C T
Raman spectroscopy is used to investigate magnetite nanoparticles dispersed in two types of $\beta$ cyclodextrin suspensions. An approach is presented for characterization of the magnetic core in liquid surrounding at room temperature and atmospheric pressure. The effect of elevating laser power on the structural stability and chemical composition of magnetite in the ferrofluids is discussed. The data are compared with data from dry by-products from the fluids. Powder samples undergo total phase transition from magnetite to hematite at laser power of $1.95 \mathrm{~mW}$. The same nanoparticles in the fluid undergo transformation at $9 \mathrm{~mW}$, but no hematite positions appear throughout that investigation. The Raman spectra revealed that the main phase of the magnetic core in the fluids is magnetite. That is indicated by a strong and non-diminishing in intensity peak at $670 \mathrm{~cm}^{-1}$. A second phase is present at the nanoparticle's surface with Raman spectroscopy unveiling maghemite-like and small fractions of goethite-like structures. The Fourier transform infrared spectroscopy investigations confirm deviations in the surface structure and also point to the fact that the oxidation process starts at an early stage after formation of the nanoparticles. The analyses of the infrared data also show that $\beta$-cyclodextrin molecules retain their cyclic character and the coating does not affect the oxidation process once the particles are evicted from the fluids. A Mössbauer spectroscopy measurement on a ferrofluidic sample is also presented.
\end{abstract}

(c) 2010 Elsevier B.V. All rights reserved.

\section{Introduction}

Since the first magnetic nanoparticles necessary to prepare a stable ferrofluid were prepared in the early 1960s [1]; they have found many interesting applications in fields like sealing, damping, heat transfer, loudspeakers, measuring devices, etc. [2]. Since then, the advances in synthesis technologies of the main magnetic component used in these ferrofluids - iron oxide, and in the methods of its analysis, had opened new wide possibilities for their application in the field of medicine [3]. Nowadays ferrofluids are extensively studied for their potential role as both diagnostic and cancer therapeutic agents [4]. This also includes studies on magnetic drug delivery systems [3], magnetic fluid hyperthermia [5], and as contrast agents for magnetic resonance imaging (MRI) [6], with currently nearly $50 \%$ of the advocated $T_{2}$-agents in MRI being based on ferrofluids containing superparamagnetic

\footnotetext{
* Corresponding author at: Institute of Electronics, Microwave Magnetics, BAS, 72, Tzarigradsko Chaussee, 1784 Sofia, Bulgaria. Tel.: +359 297958 63; fax: +35929753201.

E-mail address: 1_slavov@ie.bas.bg (L. Slavov).
}

magnetite nanoparticles [7]. In all cases, the magnetic fluids used are based on superparamagnetic iron oxide nanoparticles (magnetite or maghemite) dispersed in water. Their low toxicity and suitable magnetic properties - high relaxation signal of the particles used as a contrast agent and high magnetic moment of the particles used for drugs targeting, determine the choice of these magnetic materials. But unlike the composite, which is a dry magnetic material, the chemical and structural characterization of the iron oxide particles while in liquid medium is a difficult task, due to the evident constraints of the most applied measurement techniques.

Raman spectroscopy allows characterization of many types of samples without any specific preparation [8], which encouraged our experiments with liquids. In this paper, we focus on Raman micro-spectroscopy investigation of magnetite nanoparticles dispersed in two types of ferrofluids. Contributions to Raman spectroscopy investigations of nanosized iron oxides have already been reported, with some special emphasis on the works of da Faria et al. [9] and Shebanova and Lazor [10]. Some ferrofluid investigations involving this vibrational spectroscopy have also been made, where the preparative techniques yield either dried [11], precipitated [12], or frozen [13] samples to be measured. In 
our investigations, we used a simple procedure to probe the state of the magnetic core in liquid surrounding at normal conditions.

The aim of the investigation was to obtain new information about the development towards a second, maghemite-like phase on the surface of magnetite nanoparticles [14], when dispersed in liquid using Raman spectroscopy. The role of the liquid surrounding for reducing or preventing such development was also studied. Since all ferrofluids are known to be polydisperse to some degree [5], structural deviations on the magnetic nanoparticle's surface, if present, will complicate even more the choice of exact parameters of the applied external magnetic field. FTIR (Fourier transform infrared spectroscopy) measurements on powder samples from the fluids are also presented. Mössbauer spectroscopy was performed on specially prepared sample to probe the material as closely as possible to its fluid state. The synthesis routine of the ferrofluids is also described, since, to the best of our knowledge, only two other groups are involved in combining magnetite with beta-cyclodextrin $(\beta-C D)[15,16]-a$ water soluble cyclic oligosaccharide, used in many commercial pharmaceutical products $[17,18]$.

\section{Synthesis}

Using different approaches for magnetite stabilization, two general types of ferrofluids were prepared. The first type (type A) was based on hybrid coating of TMAOH/ $\beta-\mathrm{CD}$ on magnetite superparamagnetic particles, where the tetramethylammonium hydroxide - $\mathrm{N}\left(\mathrm{CH}_{3}\right)_{4} \mathrm{OH}$, was the surfactant. Some investigations were performed on this type of ferrofluids - for MRI response [19] and for hyperthermia effect [20]. In the second type of ferrofluids (type $B$ ), the coating was solely of $\beta-C D$. The difference between them is elimination of the surfactant from the process of particle stabilization. In such way, the cyclodextrin capability to absorb onto magnetite surface can be assessed and compared with the surfactant specifically designed for that purpose. For both types, magnetite was obtained via a co-precipitation routine described elsewhere [21].

For fluids type A (A-MFs), magnetic material with high water content was transferred into $0.2 \mathrm{ml}$ of TMAOH, homogenized, and subsequently diluted with water solution of $\beta$-cyclodextrin (Table 1). For fluids type B (B-MFs), the magnetic material obtained was transferred directly into the $\beta$-cyclodextrin solution. For all ferrofluid samples, we used $10^{-2} \mathrm{M}$ water solution of $\beta$-cyclodextrin. All experiments were conducted at atmospheric pressure and room temperature. Some initial precipitation in B-MFs was used to obtain composite samples for measurements. Minor precipitation was observed in the AMFs. After 3-day period, all fluids were separated from the sediment fraction; and the stability period observed is given in Table 1 . The powder samples subject to measurements in this paper were obtained as follows:
- Sample MAG is magnetite, precursor for all ferrofluids, dried at $70{ }^{\circ} \mathrm{C}$ for $24 \mathrm{~h}$.

- Samples pB1, pB2, and pB3 are the precipitates dried at $70{ }^{\circ} \mathrm{C}$ for $24 \mathrm{~h}$, respectively, from B1, B2, and B3 (Table 1 ).

Due to the small quantity of sedimentation, such dry samples cannot be obtained non-aggressively from the A-MFs.

\section{Results and discussions}

The Raman spectra were obtained using a LabRAM HR visible single spectrometer equipped by a microscope and a Peltiercooled CCD detector. The $633 \mathrm{~nm}$ He-Ne laser line was used for excitation. The power was adjusted using a set of neutral filters. The spectral slit width at the conditions used was $1 \mathrm{~cm}^{-1}$. In our investigations, around $3 \mathrm{ml}$ from each ferrofluid sample were placed by a pipette in an open, homemade, container of Al-foil. The laser beam was focused on the fluid surface by an X20 long working distance objective. The acquisition time for all liquid samples in Fig. 1 was $60 \mathrm{~s}$ ( $\times 2$ - times per scan) for all optical excitation intensities. This time was chosen in order to minimize the drop of level due to evaporation and at the same time to get signal sufficient for analyses from the nanosized magnetic core. For powder samples, the laser beam was focused on a flat surface by an X100 objective with $150 \mathrm{~s}(\times 2)$-acquisition time. The Raman measurements were performed at room temperature and atmospheric pressure.

By raising the laser power from 0.9 to $9.0 \mathrm{~mW}$, we tested the magnetite structural stability in the fluids. The characteristic peak positions of magnetite $\left(\mathrm{Fe}_{3} \mathrm{O}_{4}\right)$ and its possible oxidation byproducts, maghemite $\left(\gamma-\mathrm{Fe}_{2} \mathrm{O}_{3}\right)$ and hematite $\left(\alpha-\mathrm{Fe}_{2} \mathrm{O}_{3}\right)$ determined the Raman region of interest in this investigation: 100$1200 \mathrm{~cm}^{-1}$. In all spectra in Fig. 1, we include a spectrum of $\beta$ cyclodextrin, crystallized from $10^{-2} \mathrm{M}$ water suspension, since it is present in every sample measured. Its most intense peak positions are shown in Table 2. Also in Table 2 are the wavenumbers for the strongest peaks found in all ferrofluid samples at different laser excitation intensities.

For correct assignment of the band positions present in our samples and for phase identification, we used combined Raman data for key iron oxides bands $[8,9,11,22]$, from where:

- $\mathrm{Fe}_{3} \mathrm{O}_{4}$ : 193 (week), 306 (week), 538 (week), 668 (strong);

- $\gamma-\mathrm{Fe}_{2} \mathrm{O}_{3}$ : 350 (strong), 500 (strong), 700 (strong); and

- $\alpha-\mathrm{Fe}_{2} \mathrm{O}_{3}$ : 225 (strong), 247 (week), 299 (strong), 412 (strong), 497 (week), 613 (medium).

The surfactant assisted A-MFs showed very good colloidal stability. The TMAOH concentration in these fluids is not sufficient for detection and will not be discussed. Figs. 1(a) and (b) show the Raman spectra of the A-MFs with stepwise increase of the laser power. At $0.90 \mathrm{~mW}$ in both samples, a single broad

Table 1

Synthesis and colloidal stability data for the samples investigated.

\begin{tabular}{|c|c|c|c|c|c|}
\hline $\begin{array}{l}\text { Ferrofluid } \\
\text { sample }\end{array}$ & $\begin{array}{l}\text { Surfactant } \\
\text { TMAOH }(\mathrm{ml})\end{array}$ & $\begin{array}{l}\text { Concentration } \boldsymbol{\beta} \text { - } \\
\text { cyclodextrin/ } / \mathbf{H}_{2} \mathbf{O}(\mathrm{mg} / \mathrm{ml})\end{array}$ & $\begin{array}{l}\text { Concentration } \mathrm{Fe}_{3} \mathrm{O}_{4} / \\
\text { solution }(\mathrm{mg} / \mathrm{ml})\end{array}$ & $\begin{array}{l}\text { Precipitated fraction } \\
(\%)^{\mathrm{b}}\end{array}$ & $\begin{array}{l}\text { Colloidal stability } \\
\text { (days) }\end{array}$ \\
\hline A1 & 0.2 & 11 & 3 & Minor & 90 \\
\hline A2 & 0.2 & 11 & 4 & Minor & 90 \\
\hline B1 & No & 11 & $0.6^{\mathrm{a}}$ & 18 (sample pB1) & 60 \\
\hline B2 & No & 11 & $1.5^{\mathrm{a}}$ & 18 (sample pB2) & 60 \\
\hline B3 & No & 11 & $2.5^{\mathrm{a}}$ & 16 (sample pB3) & 60 \\
\hline
\end{tabular}

\footnotetext{
a Recalculated taking into account the percentage of magnetite fraction precipitated.

${ }^{\mathrm{b}}$ Relative to the initial magnetite concentration.
} 
a

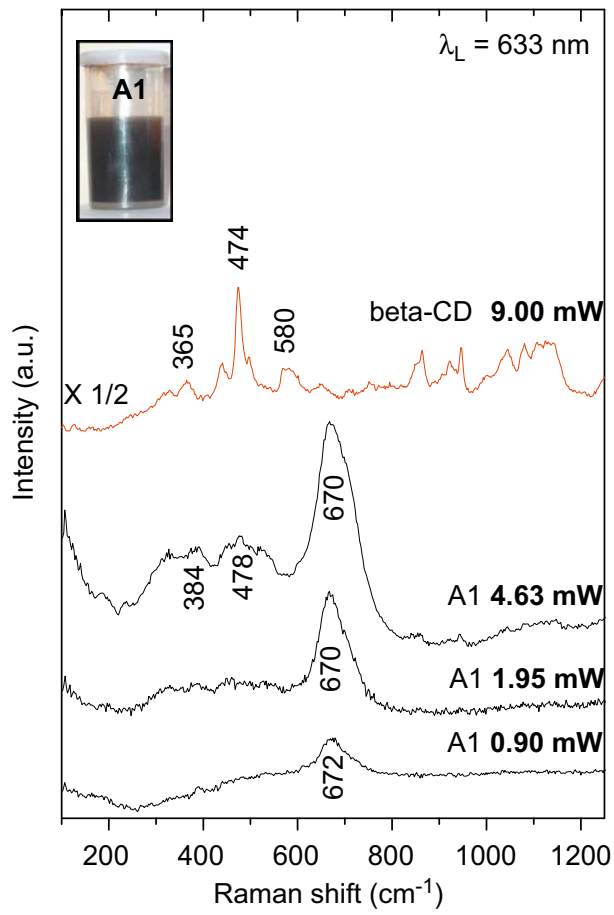

C

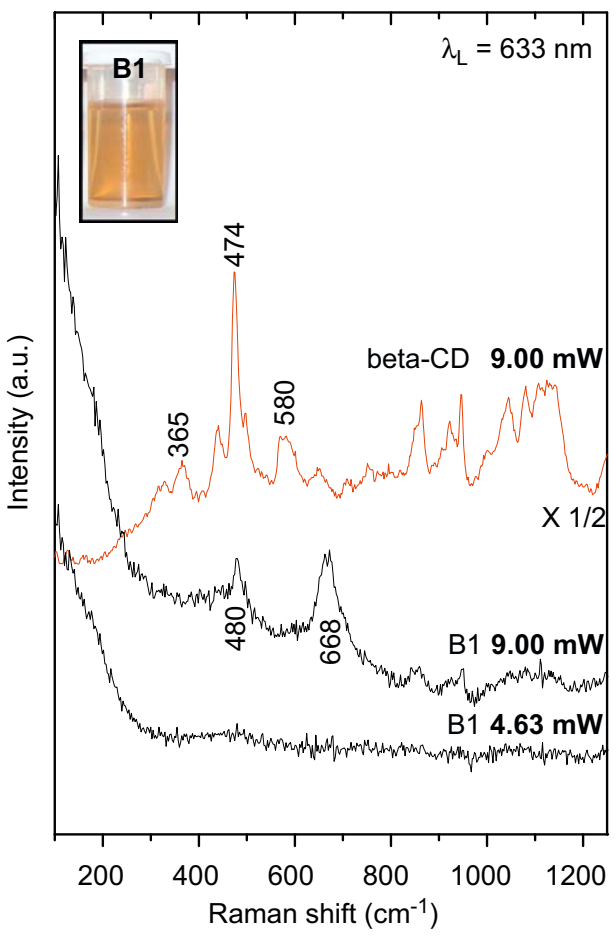

d

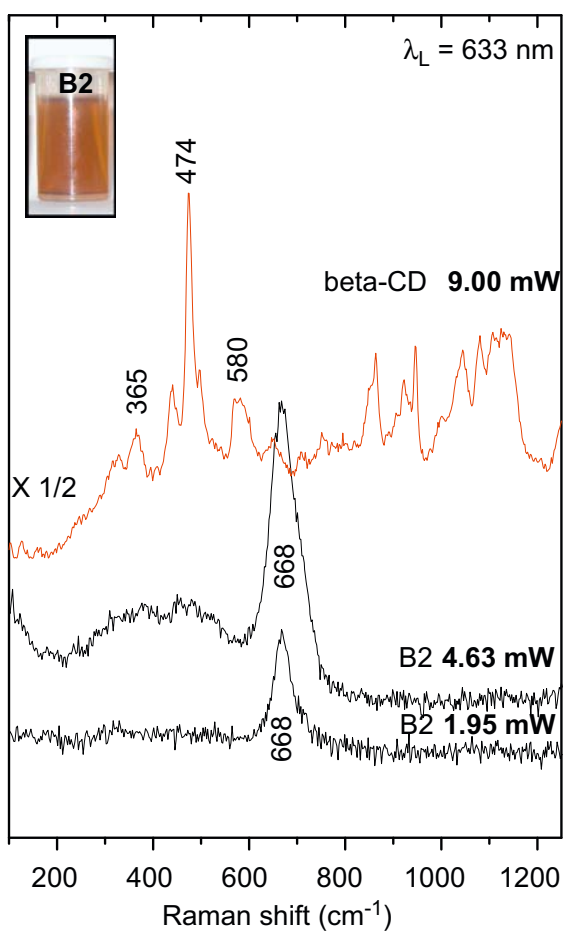

b

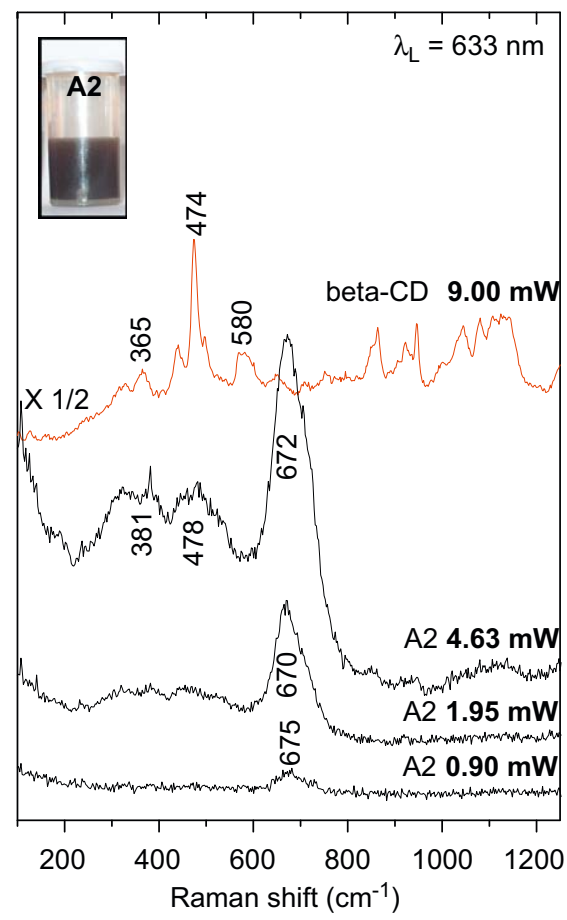

$\mathrm{e}$

Fig. 1. Raman spectra of magnetic fluids: (a) A1; (b) A2; (c) B1; (d) B2, and (e) B3.

structure at around $670 \mathrm{~cm}^{-1}$ emerges. Although this band position matches exactly that of magnetite's strongest peak, it should be dealt with caution and analyzed together with other spectral regions [8]. The absence of other spectral features in the spectra, including the low intensity peaks of magnetite, we ascribe to the low concentration and the small size (below $10 \mathrm{~nm}$, data in [20]) of the magnetic particles in the ferrofluids and to the low laser power.
Increasing the laser power to $1.95 \mathrm{~mW}$ leads to the appearance of a very broad and poorly resolved feature starting from 250 to $580 \mathrm{~cm}^{-1}$ in both samples. Ascribing exact positions to that feature of any iron oxide or hydroxide having peaks in that region will be speculative at this point. Also in that region are some of the $\beta$-cyclodextrin strongest peaks fall also in this region, and an overall overlapping could be a possible explanation for it. The peak at $670 \mathrm{~cm}^{-1}$ retains its width and significantly increases in intensity. Laser induced transformation into hematite was not 
Table 2

The position of the most intensive lines in the Raman spectra of the samples investigated.

\begin{tabular}{|c|c|c|c|c|c|c|}
\hline \multirow[t]{3}{*}{ Laser power (mw) } & \multicolumn{6}{|l|}{ Sample } \\
\hline & A1 & A2 & B1 & B2 & B3 & $\beta$-Cyclodextrin \\
\hline & \multicolumn{6}{|l|}{ Raman shift $\left(\mathrm{cm}^{-1}\right)$} \\
\hline $0.90(1.11)$ & 672 & 675 & - & - & - & \\
\hline 1.95 & 670 & 670 & - & 668 & 668 & \\
\hline \multirow[t]{3}{*}{4.63} & 670 & 672 & - & 668 & 668 & \\
\hline & 478 & 478 & - & - & 470 & \\
\hline & 384 & 381 & - & - & - & \\
\hline \multirow[t]{5}{*}{9.00} & Degradation of sample & Degradation of sample & 668 & Degradation of sample ${ }^{a}$ & Degradation of sample & 365 \\
\hline & & & 480 & & & 474 \\
\hline & & & & & & 580 \\
\hline & & & & & & 863 \\
\hline & & & & & & 946 \\
\hline
\end{tabular}

a See Fig. 2.

observed, with its strongest peaks at 225 and $246 \mathrm{~cm}^{-1}$ absent from both A-MFs spectra.

At $4.63 \mathrm{~mW}$ laser power, the most intense band remains at around $670 \mathrm{~cm}^{-1}$ in both A-MFs spectra. The small shift observed $\left(2 \mathrm{~cm}^{-1}\right)$ towards higher frequencies for $\mathrm{A} 2$ ferrofluid is in the frequency error range. The close frequencies of the $670 \mathrm{~cm}^{-1}$ line in the spectra obtained at different laser power show that the overheating of the samples on the spot investigated is minimal. A very low in intensity feature at $193 \mathrm{~cm}^{-1}$ could be noticed in both spectra, assigned by Shebanova to be characteristic for magnetite [22]. The weak contribution in the spectra between 250 to $580 \mathrm{~cm}^{-1}$ emerged at $1.95 \mathrm{~mW}$, is now well resolved. Also some very weak features at 854 and $943 \mathrm{~cm}^{-1}$ could be seen. These low-intensity peaks we assign to characteristic $\beta$-cyclodextrin band positions. Interestingly, if one compare the intensity in signal of these two weak features with the other characteristic $\beta$ cyclodextrin peaks between 250 and $600 \mathrm{~cm}^{-1}$, one should see that accept for $474 \mathrm{~cm}^{-1}$ peak, all are relatively equal in intensity. That made us believe that the $\beta$-cyclodextrin signal between 250 and $600 \mathrm{~cm}^{-1}$ region is not the main contributor, except for the strongest band position mentioned. In fact, the $474 \mathrm{~cm}^{-1}$ peak appears in all spectra (Fig. 1), at the highest laser power reached for the different samples.

With could not clearly establish hematite's strongest peak positions below $250 \mathrm{~cm}^{-1}$, its contribution to this region in the spectra should be overruled. Other possible iron oxide or hydroxide that could have contribution in this region, when considering magnetite nanoparticles, is non-stoichiometric goethite $(\alpha-\mathrm{FeOOH})[23]$ with its high intensity peak situated at $385 \mathrm{~cm}^{-1}$. The fact that we probe directly a water suspension lead us to the assumption that the $385 \mathrm{~cm}^{-1}$ contribution, seen in both $4.63 \mathrm{~mW}$ spectra, is due to some $[\mathrm{OH}]^{-}$groups linked with $\mathrm{Fe}^{3+}$ situated on magnetite's surface, resulting in non-stoichiometric goethite-like structure. On the basis of our previous investigations, we think that this structure, just a few atomic layers thick, may be finally present on what is known to be a very distorted surface of magnetite nanoparticles. The synthesis routine where magnetite is highly hydrated when mixed with solution backs that assumption. Most likely, these linked hydroxide groups exist even prior to the transfer of the magnetic particles into the solution. Other possibility for the appearance of the $385 \mathrm{~cm}^{-1}$ band is linking between $[\mathrm{OH}]^{-}$groups from the surfactant molecule with surface $\mathrm{Fe}^{3+}$ atoms of the particles. Further investigations are needed for more accurate assessment.

The absence of a shoulder around $720 \mathrm{~cm}^{-1}$ or even a splitting in both spectra at this wavenumber should not rule out the possibility of a phase transition towards maghemite. A FWHM of more than $80 \mathrm{~cm}^{-1}$ for the magnetite peak at $670 \mathrm{~cm}^{-1}$ could mean overlapping with the maghemite position at $720 \mathrm{~cm}^{-1}$. At the same time, nanosizing the material [24] could also lead to a linewidth broadening in the Raman spectra. Maghemite's other two characteristic features lie in the $250-600 \mathrm{~cm}^{-1}$ region. Overall the spectra in that region follow quite closely the maghemite pattern.

As expected, the second type of ferrofluids based on single cyclodextrin shelling showed lower colloidal stability with time compared with A-MFs. The present investigation is only the second such one carried out on such type of ferrofluids [15]. Bocanegra-Diaz et al. claimed an inclusion of magnetite particles in the $\beta$-cyclodextrin cavity in their ferrofluids. Producing magnetite particles "less than $2 \mathrm{~nm}$ " [15] in size capable of penetrating a cavity with diameter of $0.7 \mathrm{~nm}$ [25] needs some more proof; nevertheless their findings and experiment are worth mentioning.

In our ferrofluids, no assessment was made on the type of linkage between the magnetite core and the cyclodextrin molecule due to the signal change from the organic agent which was too low for Raman detection.

Figs. 1(c), (d), and (e) show Raman spectra of the B-MFs with stepwise increase of the laser power. The first Raman signals sufficient for discussion were obtained at laser power of $1.95 \mathrm{~mW}$ for B2 and B3 ferrofluids indicating strong concentration dependence. For the most diluted B1 sample, no signal was retrieved until the laser power was raised to $9 \mathrm{~mW}$.

At $1.95 \mathrm{~mW}$ in both B2 and B3 spectra, a single peak at $668 \mathrm{~cm}^{-1}$ can be seen attributed to magnetite strongest band position. Raising the power for B2 sample to $4.63 \mathrm{~mW}$ resulted in an appearance of the same as in the A-MFs poorly resolved feature starting from 250 to $580 \mathrm{~cm}^{-1}$. The discussions for the same region for A-MFs also hold true here. For B3 at this laser power, the peaks at $944,860,477 \mathrm{~cm}^{-1}$ are at the characteristic cyclodextrin positions. In fact, our assessment of these spectra is that all features in region $200-580 \mathrm{~cm}^{-1}$ are cyclodextrin related, with possible overlap of the low intensity peaks of magnetite.

The fact that the position around $670 \mathrm{~cm}^{-1}$ increases its intensity while retaining its shape in both B2 and B3 ferrofluids at $4.63 \mathrm{~mW}$ is a clear signal for a very good structural and chemical stability of the magnetite core. The development of a distorted layer onto the nanoparticle's surface is to be expected, but such a high content of the main magnetite phase in the fluids can be regarded as a surprise. Judging from the relative intensities ratio between the 670 band and the bands in region $200-580 \mathrm{~cm}^{-1}$, it 
is evident that the B-MFs have their main magnetite phase better protected compared with the A-MFs.

At the experimental conditions mentioned, laser power of $4.60 \mathrm{~mW}$ for $\lambda_{1}=633 \mathrm{~nm}$, is the most suitable for examining structural features of magnetite nanoparticles in ferrofluids for magnetic particles concentrations above $1 \mathrm{ml} / \mathrm{mg}$. At this power, the laser induced hematite contamination is zero and the intensity of the bands is sufficient for assignment.

During the measurements, the size of focus spot was not changed for the different optical excitation intensities. Nevertheless, it is possible that the magnetic particles in the focus are different for every separate measurement due to motion induced by local laser heating. Since the position around $670 \mathrm{~cm}^{-1}$ characteristic only for magnetite peak is always present even at $4.63 \mathrm{~mW}$ laser power and the only changes seen are in its relative intensity, one could consider all investigations as a confirmation of magnetite particles' very good stoichiometric homogeneity in the ferrofluids. However, our optical observations during the experiments made us believe that the heat induced by the laser power dissipates through an increase in the particle's fluctuations and a subsequent transfer to water molecules. The end result is gradual evaporation of the samples. An evidence for such an assumption could be found in Fig. 2. It can be seen that all the features in the picture (i.e. air bubbles on the surface) retain relative distance among themselves. A drop in the solution level is also evident when comparing the background of the two pictures. The degradation of the sample (spot A) when exposed to $9 \mathrm{~mW}$ laser power resulted from the separation of the organic layer from the magnetic core. The spectra obtained contain a strong background without well-resolved characteristic lines.

Fig. 3 shows Raman spectra of powder samples at different optical excitation intensities. In Fig. 3(a), the starting laser power was set below $0.1 \mathrm{~mW}$. The samples from B-MFs showed only one well-resolved peak at around $670 \mathrm{~cm}^{-1}$ characteristic for magnetite. In the MAG-sample spectrum, the band at $670 \mathrm{~cm}^{-1}$ is evidently broader indicating possible small contribution from the $720 \mathrm{~cm}^{-1}$ maghemite position. That is reinforced by the appearance of broad bands near its other two characteristic features. Again, as in the A-MFs spectra at $4.63 \mathrm{~mW}$, a second in intensity peak at $383 \mathrm{~cm}^{-1}$ appears. Due to the fact that the sample was never in contact with organic agents, this peak could only result from $[\mathrm{OH}]^{-}$groups linked to the surface of magnetite particles during synthesis. An increase of power to $1.95 \mathrm{~mW}$ caused a total transformation in the Raman spectra of all samples (Fig. 3b). The $670 \mathrm{~cm}^{-1}$ position vanishes completely. The spectra obtained represent pure hematite phase with a shift to lower wavenumbers for all peaks. The shift spans between $10 \mathrm{~cm}^{-1}$ for the lowest peak position to $40 \mathrm{~cm}^{-1}$ for the two-magnon scattering feature at $1320 \mathrm{~cm}^{-1}$. These shifts are temperature dependent and in good agreement with those observed by Shebanova and Lazor [10]. Also there, a laser power of 2-3 mW had no effect on a magnetite sample with average size of particles $0.6 \mu \mathrm{m}$, whereas in our experiment $1.95 \mathrm{~mW}$ produced complete phase transition for our nanosized samples. A direct connection between the particle size and the laser power could be drawn with additional calculations needed.

When comparing spectra from nanoparticles in fluid (Fig. 1) and those from powder samples (Fig. 3), one can see that the phase transition in the dry ones takes place at much lower laser power. Although that is expected due to better heat dissipation in the fluids, the absence of any hematite positions in the ferrofluids spectra prior degradation at $9 \mathrm{~mW}$, cut mean better structural stability of the dispersed nanoparticles.

The $\beta$-cyclodextrin coating had no effect in reducing the overheating and the phase transition induced by the laser power. The spectra obtained from the coated samples and from the uncoated magnetite are absolutely identical (Fig. 3b). With the characteristic peak positions of $\beta$-cyclodextrin missing from the Raman spectra of powdered samples, another vibrational spectroscopy was used to confirm coating.

The investigation by FTIR-spectroscopy was made on a Nicolet320 FTIR spectrometer in the $4000-400 \mathrm{~cm}^{-1}$ region using the $\mathrm{KBr}$ pellet technique. The IR-spectroscopy is a useful tool for examining not only the molecular structure of the organic agent, but also the phase transition of magnetite towards maghemite $(\gamma$ $\mathrm{Fe}_{2} \mathrm{O}_{3}$ ). Magnetite has only two absorption bands [26], one at $580 \mathrm{~cm}^{-1}$ corresponding to $v(\mathrm{Fe}-\mathrm{O})$ stretching vibration in tetrahedral site, and one at around $430 \mathrm{~cm}^{-1}$ corresponding to $\delta(\mathrm{Fe}-\mathrm{O})$ torsional vibration mode of $\mathrm{Fe}$ in octahedral site. The appearance of a shoulder in the spectrum at $630 \mathrm{~cm}^{-1}$ is a clear a

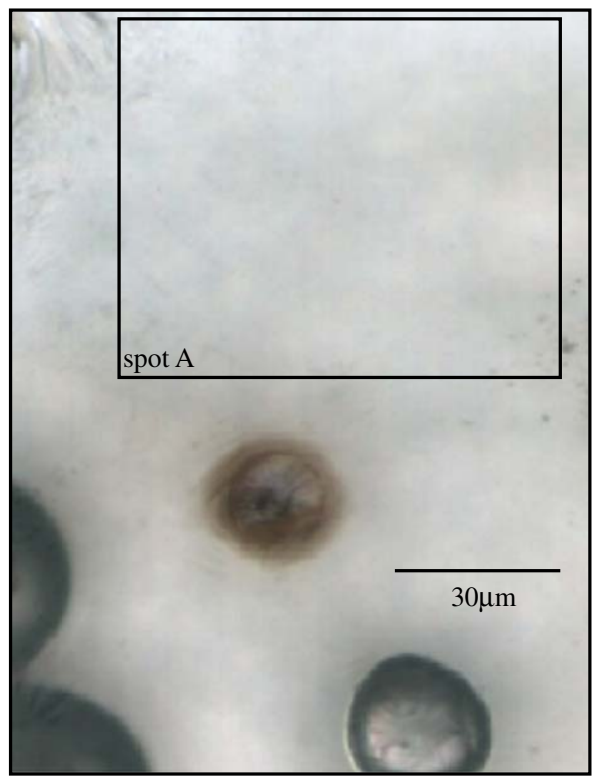

b

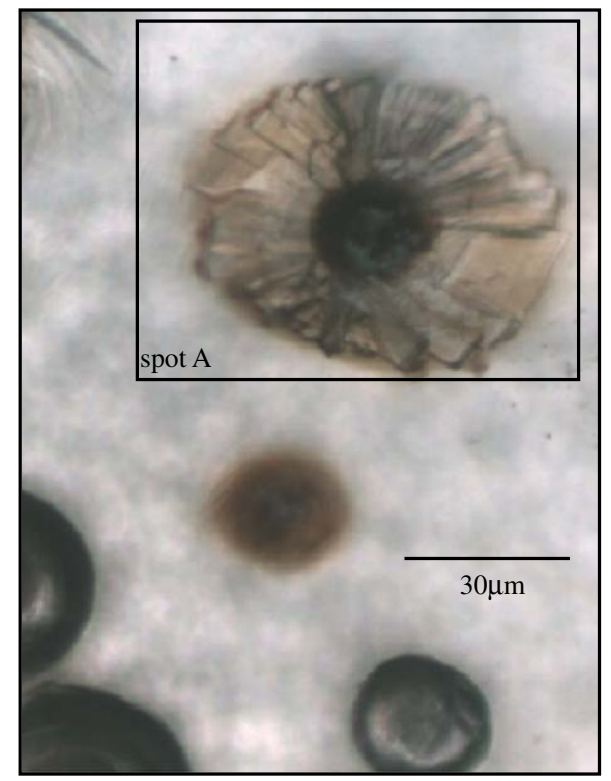

Fig. 2. Microscope image: effect of laser power on the surface of B2 ferrofluid (a) before (b) after $60 \mathrm{~s}$ irradiation with 9 mW laser power 
a

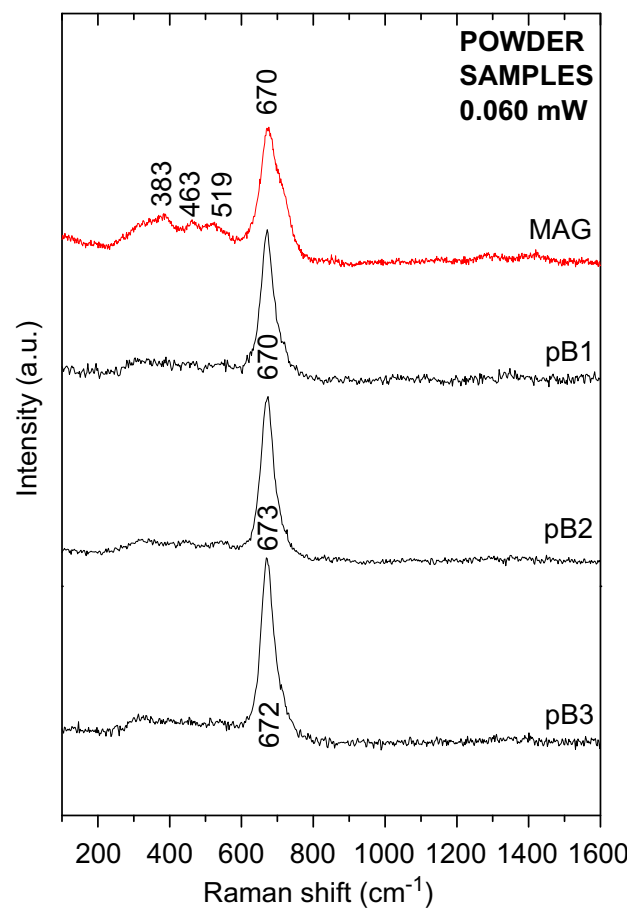

b

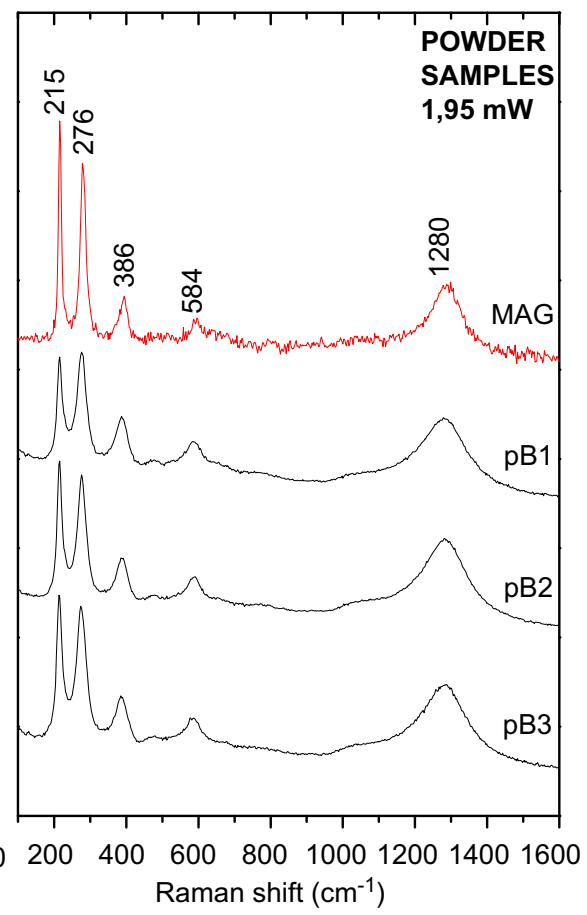

Fig. 3. Raman spectra of powdered magnetite nanoparticles at (a) $0.060 \mathrm{~mW}$ laser power and (b) $1.95 \mathrm{~mW}$ laser power.

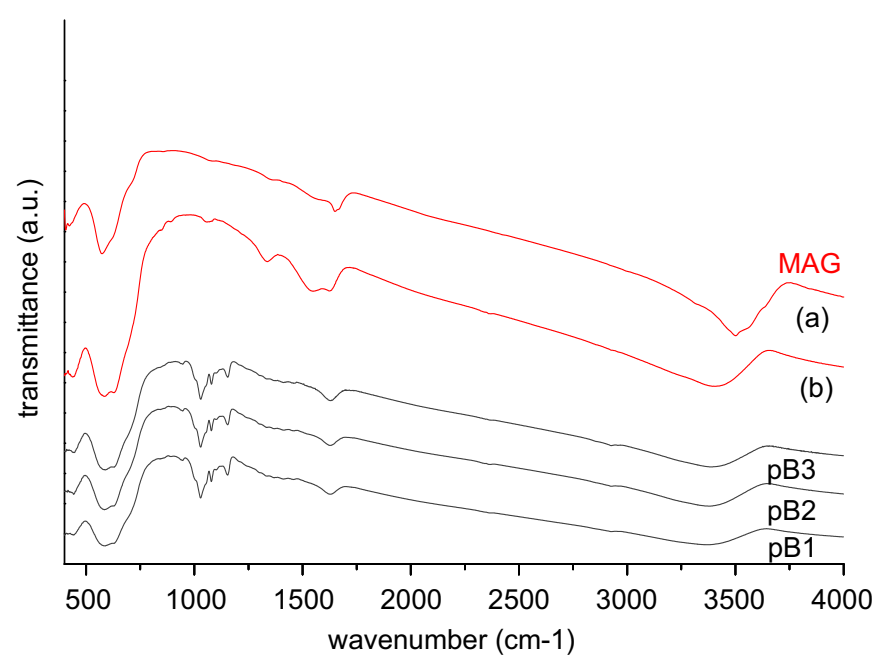

Fig. 4. FTIR spectra of powdered magnetite.

indication for a $\gamma-\mathrm{Fe}_{2} \mathrm{O}_{3}$ transition [27]. Fig. 4 presents FTIR spectra of samples precipitated from B-MFs (pB1-3) a month after drying, and of uncoated magnetite particles a week (MAG (a)) and a month after synthesis (MAG (b)).

The analysis of all spectra reveals the destructive influence of the atmospheric oxygen on the structural stability of even coated magnetite nanoparticles. For the uncoated magnetite particles, a timeline of less than 30 days meant a total transition towards maghemite. This is indicated by the appearance of a band in MAGsample (b) spectra at $630 \mathrm{~cm}^{-1}$, not observed in the same sample (a) spectra. The oxidation process starts at a very early stage after formation of the nanoparticles, which reinforces our synthesis decision for their quick transfer into a liquid medium.

All characteristic absorption bands for the organic agent are situated between 850 and $1250 \mathrm{~cm}^{-1}$ with individual assign- ments in [25]. The cyclic character of the cyclodextrin molecule is retained $[25,15]$. Also, the $\delta(\mathrm{HOH})$ vibration mode at $1650 \mathrm{~cm}^{-1}$ of water molecules existing in the cavities is present in all samples from fluids. These two facts are essential in order to use this unique structure for drug delivery applications. The absorption bands observed for the particles covered by cyclodextrin in the $\mathrm{Fe}-\mathrm{O}$ vibration region are identical with those not coated. Again, a shoulder at $630 \mathrm{~cm}^{-1}$ is evident meaning coating does not effect the oxidation process.

In our case, the band appeared around $3400 \mathrm{~cm}^{-1}$ is assigned to $v(\mathrm{O}-\mathrm{H})$ vibration mode from our samples taking up water during preparation for measurement [28]. That is because there is no difference in that region in the spectra between samples been in contact with cyclodextrin and those, which were not.

For in-vivo medical applications, the use of particles that present superparamagnetic behavior at room temperature (no remanence along with a rapidly changing magnetic state) is preferred. Using Mössbauer spectroscopy we have made an attempt to probe if there are changes in the superparamagnetic character of the particles, once they were suspended in fluids, possibly due to agglomeration. Due to some specifics in the preparation technique the data from the Mössbauer measurement presented (Fig. 5), should be regarded as informative, rather than conclusive. To confirm the superparamagnetic behavior at room temperature, ZFC FC measurements and magnetisation vs. magnetic field $(M(H))$ loops at RT should be made.

The sample under investigation (spectra in Fig. 5) was nonaggressively dried on Al-foil, at RT and atmospheric pressure, part of $\mathbf{A 1}$ ferrofluid. The holder was filled with roughly less than $1 \mathrm{ml} /$ day for 1 week in order to obtain iron content sufficient for measurement. Although time consuming, this preparative approach allowed us to probe the sample at room temperature and more importantly, it gave us the opportunity to probe the material as closely as possible to its fluid state.

The RT spectrum exhibits mainly superparamagnetic magnetite/maghemite (68\%). There is still some transition spectrum 

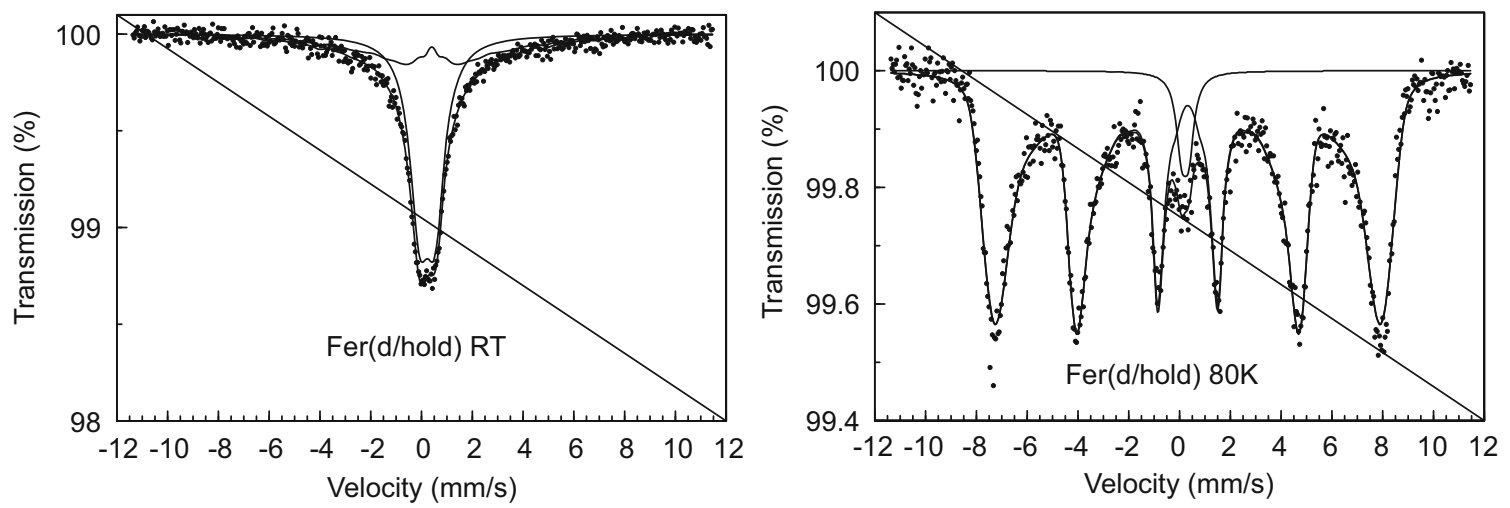

Fig. 5. Mössbauer spectra (RT and $80 \mathrm{~K}$ ) of magnetite from A1 fluid.

Table 3

Hyperfine parameters.

\begin{tabular}{|c|c|c|c|c|c|c|}
\hline Temp & $H_{\mathrm{p}}\left(H_{\mathrm{av}}\right)(\mathrm{T})$ & $2 \varepsilon_{\mathrm{Q}}(\mathrm{mm} / \mathrm{s})$ & $\delta_{\mathrm{Fe}}(\mathrm{mm} / \mathrm{s})$ & $\Delta E_{\mathrm{Q}}(\mathrm{mm} / \mathrm{s})$ & $\mathrm{RA}(\%)$ & Assignment \\
\hline \multirow[t]{2}{*}{ RT } & $9.7(2.0)$ & 0 & 0.5 & - & 32 & Transition spectr. magn/magh \\
\hline & - & - & 0.34 & 0.6 & 68 & SP magn/magh \\
\hline \multirow[t]{2}{*}{$80 \mathrm{~K}$} & $47.1(41.4)$ & 0 & 0.43 & - & 95 & FM magnetite/maghemite \\
\hline & & & 0.34 & 0.3 & 5 & Fe in aluminum foil \\
\hline
\end{tabular}

present (32\%), which is due to the distribution of sizes, meaning agglomeration has taken place in the fluid. Such distribution could lead to small remanence in a RT-hysteresis loop due to the fact that the total magnetisation of the system can be expressed as $M(H)=M_{\text {superparamagnetic }}(H)+M_{\text {non-superparamagnetic }}(H)$ [29]. On the other hand, if the superparamagnetic particles are indeed covered by a distorted magnetically layer, the magnetic core/shell coupling will also contribute to the absence of a non-hysteresis loop. The organic cover, which is known to be a factor in reducing the saturation magnetisation of the system, will also play a role in a magnetic measurement [29]. From this RT-Mössbauer spectrum, it cannot be decided whether the sample contains an iron oxide closer to magnetite or closer to maghemite, nor if it is a case of magnetite core with thin layer of maghemite-like structure. For better analyses, the interaction between surface and core should be examined by studying the system in the pre-oxidized state if possible, which is a very difficult preparative task for such small particles, or to plot a hysteresis loop post field cooling at low temperatures $(5 \mathrm{~K})$. Another possibility to clarify if there is a layer with different magnetic anisotropy onto their surface is ILEEMS measurement [30]. Here, the $80 \mathrm{~K}$ spectrum is fitted with one sextet. Usually, the $80 \mathrm{~K}$ spectrum for magnetite-like and for maghemite-like small particles is very similar. The central part may arise from Fe in the aluminum foil, which has a large effect in this spectrum due to the still low concentration of the sample. Another possibility is that this central part of the $80 \mathrm{~K}$ spectrum is due to some superparamagnetic magnetite, reinforced by the isomer shift value of $0.34 \mathrm{~mm} / \mathrm{s}$ which is typical for $\mathrm{Fe}^{3+}$ (see Table 3 ) and not for iron clusters in the foil.

In our previous investigation based on Mössbauer measurements, we showed that the surface of magnetite nanoparticles has a defective structure spanning around $30 \%$ of its volume $[31,14]$. The investigations made in this paper reinforce this assumption with the Raman micro-spectroscopy being able to distinguish clearly a stoichiometric magnetite as a main phase in the ferrofluids. Although the Mössbauer measurement presented reveals that $68 \%$ of the particles are in superparamagnetic state, further FC, ZFC, $M(H)$ and ILEEMS investigations are under way in order to assess the influence of cover factors on the magnetic properties of the whole system.

\section{Summary and conclusions}

A new approach is presented for direct probing the structural stability and chemical composition of magnetite core in a ferrofluid via Raman micro-spectroscopy. Using an open homemade Al-foil container, with the laser beam focused on the fluid surface, we were able to detect a signal with intensity sufficient for assignment. We found that laser power of $4.60 \mathrm{~mW}$ for $\lambda_{\mathrm{L}}=633 \mathrm{~nm}$ is the most suitable for examining structural features of magnetite nanoparticles in ferrofluids without laser-induced hematite contamination.

The surfactant assisted A-MFs showed better colloidal stability compared with the B-MFs. The Raman spectra collected revealed the main phase in both types of ferrofluids to be magnetite. This is indicated by a very strong and non-diminishing in intensity peak at $670 \mathrm{~cm}^{-1}$. No contamination with laser induced hematite is observed even at $4.63 \mathrm{~mW}$ laser power. This is attributed to the experimental conditions. The broad features below $600 \mathrm{~cm}^{-1}$ that emerged at this power are attributed to overlap signals from $\beta$ cyclodextrin, a maghemite-like structure and small fractions of a goethite-like structure (peak at $385 \mathrm{~cm}^{-1}$ ). These two ironcontaining phases are most probably located on the surface of a stoichiometric magnetite core. If so, such surface deviations will have an influence on the ferrofluids magnetic behavior when exposed to external field. Our first attempt to assessing the magnetic character of magnetite particles in ferrofluids was via Mössbauer spectroscopy on specially prepared fluidic sample from A-MF. The RT-Mössbauer spectrum reveals that $68 \%$ of the particles in the fluid are in a superparamagnetic state with $32 \%$ transition spectrum found due to distribution of particle sizes.

Judging from the relative ratio in the intensities between of the $670 \mathrm{~cm}^{-1}$ band and the bands in the $200-580 \mathrm{~cm}^{-1}$ region in the Raman spectra discussed it is evident that the B-MFs has their main magnetite phase better protected compared with the A-MFs. 
Powder samples undergo total phase transition from magnetite to hematite at laser power of $1.95 \mathrm{~mW}$. Same particles in the fluids undergo transformation at $9 \mathrm{~mW}$, but no hematite positions were detected throughout the investigation.

The FTIR spectra of powder samples reveals that the $\beta$ cyclodextrin structure remains intact, but the coating does not slow the oxidation process which takes place with time.

\section{Acknowledgements}

LS gratefully acknowledge and would like to thank the World Federation of Scientists (WFS) for a scholarship. MA acknowledges the financial support under D002-167/2008 of the Bulgarian Ministry of Education and Science. The paper is supported by 10 $619 / 2009$ of the University of Chemical Technology and Metallurgy.

\section{References}

[1] R.E. Rosenswieg, Sci. Am. 247 (1982) 136-145

[2] K. Raj, B. Moskowitz, R. Casciari, J. Magn. Magn. Mater. 149 (1995) 174-180.

[3] T. Neuberger, B. Schöpf, H. Hofmann, et al., J. Magn. Magn. Mater. 293 (2005) 483-496.

[4] J.D. Byrne, T. Betancourt, L. Brannon-Peppas, Adv. Drug Deliv. Rev. 60 (2008) $1615-1626$.

[5] M. Gonzales-Weimuller, M. Zeisberger, K.M. Krishnan, J. Magn. Magn. Mater. 321 (2009) 1947-1950.

[6] N. Sun, D.-X. Chen, H.-C. Gu, X.-L. Wang, J. Magn. Magn. Mater., 10.1016/ j.jmmm.2009.04.073.

[7] Guo-Ping Yan, L. Robinson, P. Hogg, Radiography 13 (2007) e5-e19.
[8] I. Chourpa, L. Douziech-Eyrolles, L. Ngaboni-Okassa, et al., Analyst 130 (2005) 1395-1403.

[9] D.L.A. de Faria, S. Venancio Silva, M.T. de Oliveira, J. Raman Spectrosc. 28 (1997) 873-878

[10] O.N. Shebanova, P. Lazor, J. Raman Spectrosc. 34 (2003) 845-852.

[11] J.C. Rubim, M.H. Sousa, J.C.O. Silva, F.A. Tourinho, Braz. J. Phys. 31 (2001) 402-408.

[12] S.W. da Silva, T.F.O. Melo, M.A.G. Soler, et al., IEEE Trans. Magn. 39 (2003) 2645-2647.

[13] J.E. Weber, A.R. Goni, D.J. Pusiol, C. Thomsen, Phys. Rev. E 66 (2002) 021407.

[14] I. Nedkov, T. Merodiiska, L. Slavov, et al., J. Magn. Magn. Mater. 300 (2006) 358-367.

[15] A. Bocanegra-Diaz, N.D.S. Mohallem, R.D. Sinisterra, J. Braz. Chem. Soc. 14 (2003) 936-941.

[16] L.A.C. Cruz, C.A.M. Perez, H.A.M. Moreno, P.E.G. Casillas, J. Alloys Compd. 466 (2008) 330-334.

[17] J. Szejtli, Encyclopedia Nanosci. Nanotechnol. 2 (2004) 283-304.

[18] J. Szejtli, Chem. Rev. 98 (1998) 1743-1754.

[19] R. Kalionsky, T. Merodiiska, M. Dencheva-Zarkova, et al., C. R. Acad. Bulg. Sci. 60 (2007) 893-898.

[20] I. Nedkov, L. Slavov, T. Merodiiska, et al., J. Nanoparticle Res. 10 (2008) 877-880.

[21] Patent BG no. 107837/22.05.2003.

[22] O.N. Shebanova, P. Lazor, J. Solid State Chem. 174 (2003) 424-430.

[23] M.H. Sousa, F.A. Tourinho, J.C. Rubim, J. Raman Spectrosc. 31 (2000) 185-191.

[24] F.J. Owens, J. Orosz, Solid State Commun. 138 (2006) 95-98.

[25] J. Szejtli, in: Cyclodextrins and Their Inclusion Complexes, Akadémiai, Kiadó Budapest, 1982

[26] J. Mürbe, A. Rechtenbach, J. Töpfer, Mater. Chem. Phys. 110 (2008) 426-433.

[27] G.W. Poling, J. Electrochem. Soc. 116 (1968) 958-963.

[28] Li-Ying Zhang, Hong-Chen Gu, Xu-Man Wang, J. Magn. Magn. Mater. 311 (2007) 228-233.

[29] I. Nedkov, T. Merodiiska, L. Slavov, et al., in: I. Nedkov, Ph. Tailhades (Eds.) Nanoscale Magnetic Oxides and Bio-world, Heron Press, Sofia, 2004, pp. 29-37.

[30] E. De Grave, R.E. Vandenberghe, C. Dauwe, Hyperfine Interact. $161(1-4)$ (2005) 147-160.

[31] I. Nedkov, J. Optoelectron. Adv. Mater. 1 (2007) 24-29. 